\title{
Design and Research on Characteristics of a New Vibration Isolator with Quasi-zero-stiffness
}

\author{
Shi Peicheng ${ }^{1,}$, Nie Gaofa ${ }^{1, b}$ \\ ${ }^{1}$ Anhui Polytechnic University, Wuhu, 241000, China \\ ashipeicheng@126.com, bniegaofa@163.com
}

\begin{abstract}
Keywords: Vibration, Quasi-zero-stiffness, Harmonic balance, Low-frequency vibration isolation, Force transmissibility
\end{abstract}

\begin{abstract}
In order to solve the problem of low-frequency vibration isolation, a new vibration isolator with quasi-zero-stiffness characteristic was designed and the relationship among design parameters was analyzed. Dynamic behavior was calculated based on harmonic balance method and simulation of the vibration isolation performance was carried out. Thus, force transmissibility of the system was obtained. The simulations showed that the designed system had the quasi-zero-stiffness characteristics at long range, good vibration isolation performance and could solve the inherent contradictions between the low-and-ultralow frequency vibration isolation and system stiffness.
\end{abstract}

\section{Introduction}

According to vibration theory, the frequency of traditional passive vibration isolations attenuating external disturbance is $\sqrt{2}$ times more than their natural frequency [1]. For achieving vibration isolation of low-and-ultralow frequency within the scope from $0.5 \mathrm{HZ}$ to $70 \mathrm{HZ}$, these apparatuses only reduce their natural frequency. However, reducing the natural frequency means sacrificing the system stiffness [2]. The small system stiffness will contribute to increasing the static deflection of elastic components and decreasing the load bearing capacity. With the in-depth study, the isolation system with quasi-zero-stiffness characteristic turns out to possess excellent performance. The isolation system can obtain low dynamic stiffness without sacrificing static stiffness [3, 4]. There are several kinds of typical quasi-zero-stiffness structure described as following. The authors in [5] achieved the low frequency vibration isolation basing on an apparatus composed by a magnet and a spring. In [6] the authors took advantage of negative stiffness characteristics the beam under axial load bent in the longitudinal direction demonstrate and designed an apparatus with quasi-zero-stiffness. In [7] the authors designed a quasi-zero-stiffness structure assembled via parameter optimization by three linear or nonlinear springs, and conducted a series of static and dynamic analysis. However, the above several models can only realize quasi-zero-stiffness under the particular quality. In order to meet the demand for vibration isolation when the quality of the object varies, this paper puts forward a novel vibration isolation platform possessing adjustable quasi-zero-stiffness. As a kind of passive vibration isolation system, this scheme can not only reduce production cost and improve system reliability, but also solve the inherent conflict between the low-and-ultralow frequency vibration isolation and system stiffness.

\section{Design of Vibration Isolation System}

In this paper, the vibration isolation system with quasi-zero-stiffness shown in Fig. 1 (a) consists of horizontal thrust spring-7, bearing spring-8, workbench-3 and foundation-1. Rotating shaft-5 via cylindrical pin- 6 is installed on the foundation- 1 and is inscribed in the circular surface of workbench-3. The horizontal thrust spring-7 is installed in spring mounting hole on the foundation-1. The initial thrust is controlled by thrust regulator-2-adjusts the initial amount of compression of the horizontal thrust spring-7. The workbench-3 is simultaneously forced by the horizontal thrust spring-7 and the bearing spring-8. 


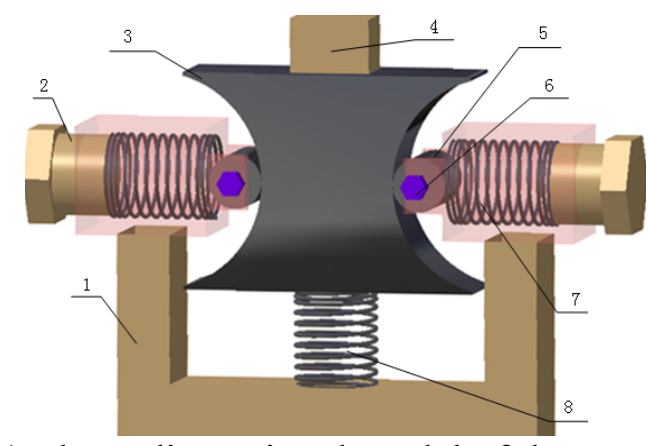

(a) Three-dimensional model of the system

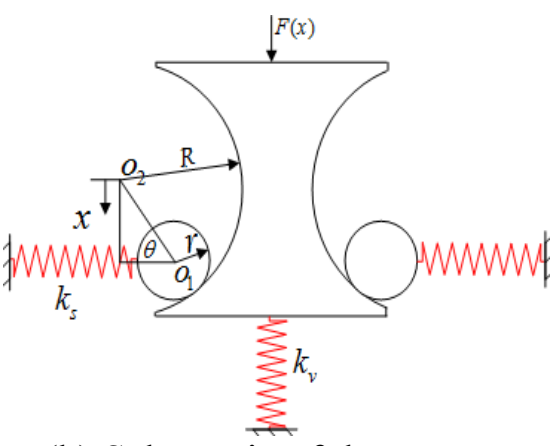

(b) Schematic of the system

1-Foundation; 2-Thrust regulator; 3-Workbench; 4-Vibration isolation object; 5-Rotating shaft; 6-Cylindrical pin; 7-Horizontal thrust spring; 8-Bearing spring.

Fig. 1 Vibration isolation platform with quasi-zero-stiffness.

\section{Static Analysis of the Proposed Design}

When the workbench has no load, Fig. 1 (b) shows the relative location between the circle $\mathrm{O}_{1}$ and the circle $\mathrm{O}_{2}$. Under the action of static force, the workbench moves downward vertically. The variable $\mathrm{x}$ is defined as the displacement deviating from the static equilibrium position shown in Fig. 1 (a).

Hypothetically, the term $F(x)$ in Fig. 1 (b) is static force. Surppose that it is equal to F, the general equation relating the force $\mathrm{F}$ to the displacement $\mathrm{x}$ in the vertical direction can be expressed as:

$$
F=k_{v} x-2 k_{s} \Delta x \tan \theta
$$

where, $\Delta x=a-\left[(R-r)-\sqrt{(R-r)^{2}-x^{2}}\right]$ and $\tan \theta=\frac{x}{\sqrt{(R-r)^{2}-x^{2}}}$

Where, the term $\theta$ in (1) is the included angle between line linking centers of the inscribed circle and central axis of the horizontal thrust spring; the term $x$ denotes amount of deformation of the horizontal thrust spring when the workbench moves nearby the equilibrium position; the term $k_{\mathrm{v}}$ denotes the stiffness of the bearing spring; the term ks denotes the stiffness of the horizontal thrust spring; the term a denotes the initial amount of compression of the horizontal thrust spring, which is adjusted by the mass that the workbench is loaded.

Then, (1) can be expressed as follows:

$$
F=k_{v} x-2 k_{s} x\left[1+\frac{a-(R-r)}{\sqrt{(R-r)^{2}-x^{2}}}\right]
$$

For clarity of analysis, the nondimensional equation rewrited from (2) whose both sides are simultaneously divided by $k_{\mathrm{v}} r$ is introduced as follows. All parameters in (3) are nondimensional.

$$
\hat{F}=\hat{x}-2 \lambda \hat{x}\left[1+\frac{\hat{a}-(\hat{R}-1)}{\sqrt{(\hat{R}-1)^{2}-\hat{x}^{2}}}\right]
$$

where, $\hat{F}=\frac{\hat{f}}{k_{v} r} ; \hat{a}=\frac{a}{r} ; \hat{R}=\frac{R}{r} ; \lambda=\frac{k_{s}}{k_{v}}$.

Eq. (3) is the expression without dimension of the force to the displacementy. To obtain stiffness formula without nondimension of the system, we take derivative of (3) with respect to $\hat{x}$. The nondimensional stiffness formula is shown in (4). 


$$
\hat{k}=1-2 \lambda\left(1+\frac{[\hat{a}-(\hat{R}-1)](\hat{R}-1)^{2}}{\left((\hat{R}-1)^{2}-\hat{x}^{2}\right)^{3 / 2}}\right)
$$

To achieve the characteristic of zero stiffness, $\hat{k}$ must be equal to 0 when $\hat{x}$ is equal to 0 . Thus, (5) can be achieved through Eq. (4).

$$
\frac{\hat{a} \lambda}{\hat{R}-1}=\frac{1}{2}
$$

When the novel platform possesses quasi-zero-stiffness, Eq. (5) denontes the relationship needed to meet among design parameters - such as stiffness ratio $\lambda$, the initial amount of compression and radius ratio $\hat{R}$.

In order to avoid the phenomenon of negative stiffness, the equilibrium position should locate the minimum point of stiffness. So we take derivative of (4) with respect to $\hat{x}$.

$$
\hat{k}^{\prime}=-\frac{6 \hat{x} \lambda[\hat{a}-(\hat{R}-1)](\hat{R}-1)^{2}}{\left((\hat{R}-1)^{2}-\hat{x}^{2}\right)^{5 / 2}}
$$

Then by substituting zero for $\hat{x}$, we can easily derive $\hat{k}^{\prime}$ is equal to zero. Eq. (6) denotes that the proposed design not only possesses reliable quasi-zero-stiffness, but also avoids passive stiffness.

Suppose that stiffness ratio $\lambda=3$, radius ratio $\hat{R}$, and the initial amount of compression $\hat{a}=0.5$, and solving (3) and (4) by Matlab, we can derive the nondimensional force-to-displacement curve and the nondimensional stiffness-to-displacement curve of this case. When the displacement of up-down vibration is within -0.5 to +0.5 , Fig. 2 and Fig. 3 respectively shows that the isolator proposed in this paper possesses raliable quasi-zero-stiffness characteristic and simultaneously illustrates that the isolator is a stable system.

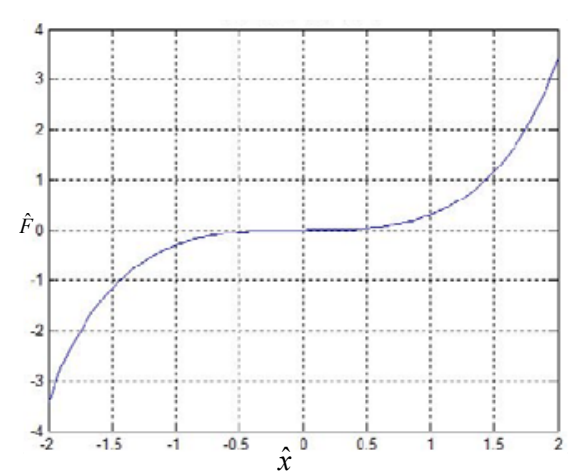

Fig. 2 Force-to-displacement curve.

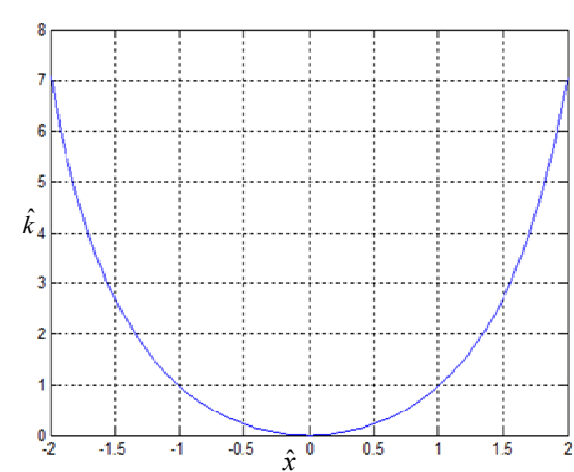

Fig. 3 Stiffness-to-displacement curve.

\section{Dynamic Analysis of the Platform}

Approximate Substitute for Restoring Force. In order to facilitate the discussion of dynamic properties of the platform, it is necessary to simplify the mathematical model. The force-to-displacement curve in Fig. 2 indicates that it is feasible to analogously substitute a cubic polynomial for the elastic restoring force of the system. By Taylor series expansion, and ignoring higher-order terms, we can derive a cubic polynomial which analogously substitutes the elastic restoring force of the proposed design.

Taylor series expansion in $\hat{x}=0$ can be expressed as:

$$
\hat{F}=\hat{F}(0)+\hat{F}^{\prime}(0) \hat{x}+\frac{\hat{F}^{\prime \prime}(0)}{2 !} \hat{x}^{2}+\frac{\hat{F}^{\prime \prime \prime}(0)}{3 !} \hat{x}^{3}+\cdots
$$

Thus, the approximate expression of force-to-displacement can be expressed as:

$$
\hat{F}=\hat{F}(0)+\hat{k}(0) \hat{x}+\frac{\hat{k}^{\prime}(0)}{2 !} \hat{x}^{2}+\frac{\hat{k}^{\prime \prime}(0)}{3 !} \hat{x}^{3}
$$

Under the conditions $\hat{F}(0)=0, \hat{k}(0)=0, \quad \hat{k}^{\prime}(0)=0$, Eq. (9) can be achieved. 


$$
\hat{F}=\frac{\hat{k}^{\prime \prime}(0)}{3 !} \hat{x}^{3}=\frac{\lambda((\hat{R}-1)-\hat{a})}{(\hat{R}-1)^{3}} \hat{x}^{3}
$$

Eq. (9) is the reduced force-to-displacement equation of the system.

Fig. 4 displays the error between approximate solution and exact solution of force-to-displacemen. Fig. 5 shows the error between approximate stiffness and exact stiffness of the stiffness-to-displacemen. In the range of small vibration amplitudes nearby the equilibrium position $\hat{x}=0$ shown in Fig. 4 , the approximate curve coincides with the accurate curve well. So analogously substituting the cubic polynomial for the elastic restoring force is feasible within the range of small vibration amplitudes nearby the equilibrium position.

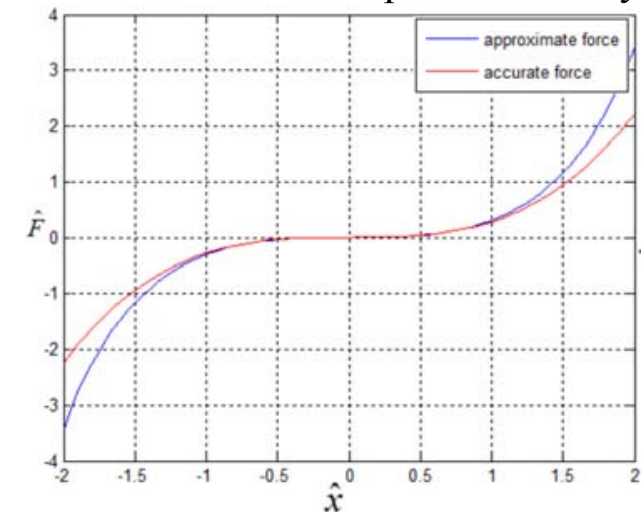

Fig. 4 Comparation between approximate force and accurate force.

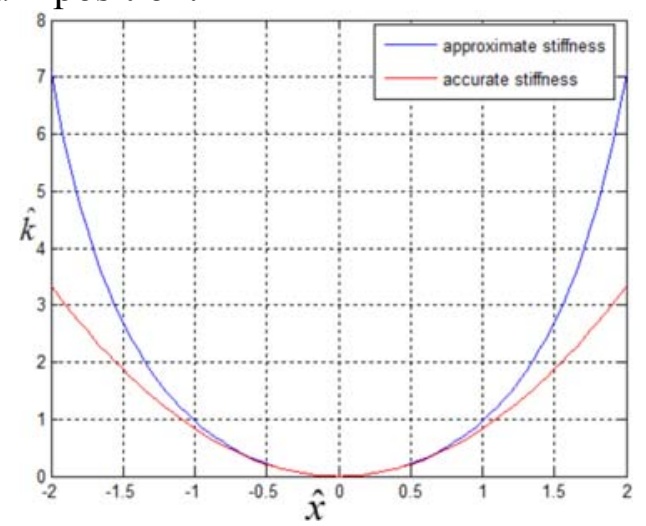

Fig. 5 Comparation between approximate stiffness and accurate stiffness.

Performance Analysis of the Vibration Isolation System. For linear vibration system, force transmissibility is often applied in studying vibration chracteristics of this kind of isolator. For this isolator with quasi-zero-stiffness characteristic, force transmissibility can be also suitable for analyzing its performance characteristic. Fig. 6 is schematic diagram of the dynamic model.
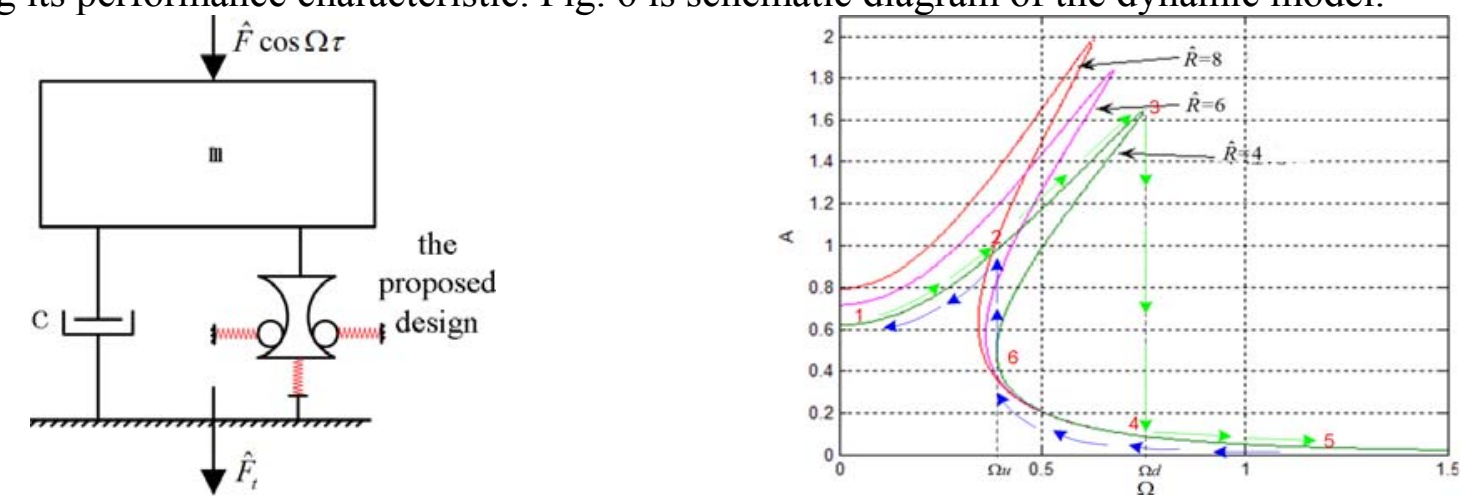

Fig. 6 Schematic diagram of the dynamic model. Fig. 7 Frequency-amplitude for $_{\zeta}=0.02, \hat{F}=0.05$

Under the influence of harmonic exciting force and damping, the nondimensional motion equation of force-to-displacement can be derived by substituting the appraximate cubic polynomial for the elastic restoring force. Actually the motion equation is Duffing equation without linear term.

$$
\hat{\ddot{x}}+2 \zeta \hat{\dot{x}}+\gamma \hat{x}^{3}=\hat{F} \cos \Omega \tau
$$

where, $\hat{x}=\frac{x}{r}, \hat{F}=\frac{F}{k_{v} r}, \omega_{0}^{2}=\frac{k}{m}, \tau=\omega_{0} t, \Omega=\frac{\omega}{\omega_{0}}, \zeta=\frac{c \omega_{0}}{2 k_{\text {QZS }}}, \gamma=\frac{\lambda((R-1)-\hat{a})}{(R-1)^{3}}$

Suppose that solution of (10) is equal to $\hat{x}(\tau)=A \sin (\Omega \tau+\theta)$. Harmonic balance method is applied to solve the motion equations of the nonlinear system. The coefficients of the same cosines and sines on both sides of (10) must be equal and ignoring high-order harmonic and eliminating phase. The frequency-amplitude formulation of displacement response to fundamental harmonic consequently can be derived, which is shown in (11).

$$
\left(\frac{3}{4} \gamma A^{3}-A \Omega^{2}\right)^{2}+(2 \zeta A \Omega)^{2}=\hat{F}^{2}
$$


In (11), $\mathrm{A}$ is the response amplitude of the system; $\Omega$ is the frequency of the harmonic excitation. Eq. (11) describes the relationship between the response amplitude A and damping ratio $\zeta$, stiffness ratio $\gamma$, and excitation condition, including the amplitude of the exciting force and exciting frequency. From $\gamma=\frac{\lambda((\hat{R}-1)-\hat{a})}{(\hat{R}-1)^{3}}$, it can be discovered that the relationship between the response amplitude $\mathrm{A}$ and stiffness ratio $\gamma$ depends on system parameters such as radius ratio $\hat{R}$. With the increase of radius ratio $\hat{R}$, stiffness ratio decreases. Fig. 7 shows the influnce of radius ratio $\hat{R}$ on the frequency-amplitude characteristics. As is shown in the graph, with the increase of radius ratio $\hat{R}$, the jump-up frequency $\Omega_{\mathrm{d}}$ and jump-down frequency $\Omega_{\mathrm{u}}$ decreases, respectively.

Multiple solutions, namely the jumping frequency domain, appear in the curves of frequency-amplitude characteristic, because of the influence of nonlinear phenomenon. Hypothetically, the constant amplitude of exciting force, as the exciting frequency changes from low to high, vibration amplitude A along the green curve goes through the point 2 after passing 1 to reach the maximum point 3 . If the exciting frequency still increases, vibration amplitude $\mathrm{A}$ abruptly jumps down to the point 4 from the maximum point 3 and finally reaches the point 5 along the green curve. On the contray, as the exciting frequency changes from high to low, amplitude A along the blue curve goes through the point 4 after passing 5 to reach the point 6 . If the exciting frequency still decreases, vibration amplitude A abruptly jumps to the point 2 in the upper branch from the point 6 and finally reaches the point 1 along the blue curve.

Suppose that $f_{t e}, f_{t d}$ is respectively the elastic force and damping force. The force $\hat{F}_{t}$ transmitted to the base from the vibration isolation can be expressed as:

$$
\hat{F}_{t}=\sqrt{f_{t e}^{2}+f_{t d}^{2}}
$$

The amplitude $\left|\hat{F}_{t}\right|$ of the force transmitted to the base can be expressed as:

$$
\left|\hat{F}_{t}\right|=\sqrt{\left(\frac{3}{4} \gamma A^{3}\right)^{2}+(2 \zeta A \Omega)^{2}}
$$

Eq. (13) denotes the relationship between the amplitude $\left|\hat{F}_{t}\right|$ of the force $\hat{F}_{t}$ and damping ratio $\zeta$, stiffness ratio $\gamma$, and excitation condition.

So force transmissibility is expressed as:

$$
T=\frac{|\hat{F}|}{\hat{F}}=\frac{\sqrt{\left(\frac{3}{4} \gamma A^{3}\right)^{2}+(2 \zeta A \Omega)^{2}}}{\hat{F}} .
$$

Eq. (14) denotes that force transmissibility of the quasic-zero-stiffness system depends on the size of damping ratio $\zeta$ and the amplitude of exciting force.

Based on (14), the dynamic response of the isolator with respect to ratio $\zeta$ can be investigated. Figure 8 shows the influence of the ratio $\zeta$ on force transmissibility. The larger values of $\zeta$ the larger isolation range will be achieved. More specifically, according to Fig. 8 , when $\zeta=0.01$, the curve obviously bends to the right and marked jumping phenomenon occurs, which leads to the increase of the initial frequency to the vibration isolation and attenuation of the effect of the vibration effect. When $\zeta=0.02$, jumping phenomenon becomes attenuated and the transmissibility is under 15 percent, especially the peak transmissibility is reduced markedly campared with that of $\zeta=0.01$. When $\zeta=0.03$, jumping phenomenon disappears thoroughly and the transmissibility is under 5 percent. And the initial frequency is the lowest among the three values of the ratio $\zeta$. 


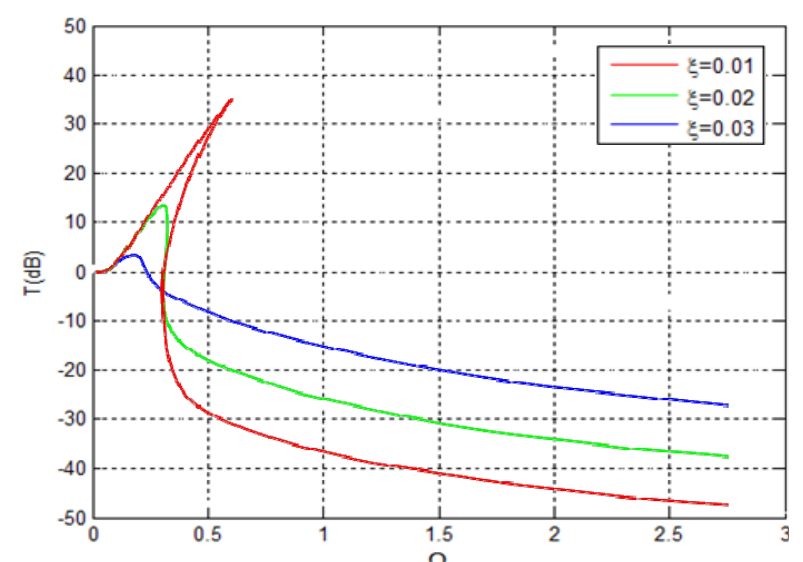

Fig. 8 Influence of $\zeta$ on force transmissibility for $\hat{F}_{t}=0.05, \gamma=5 / 18$

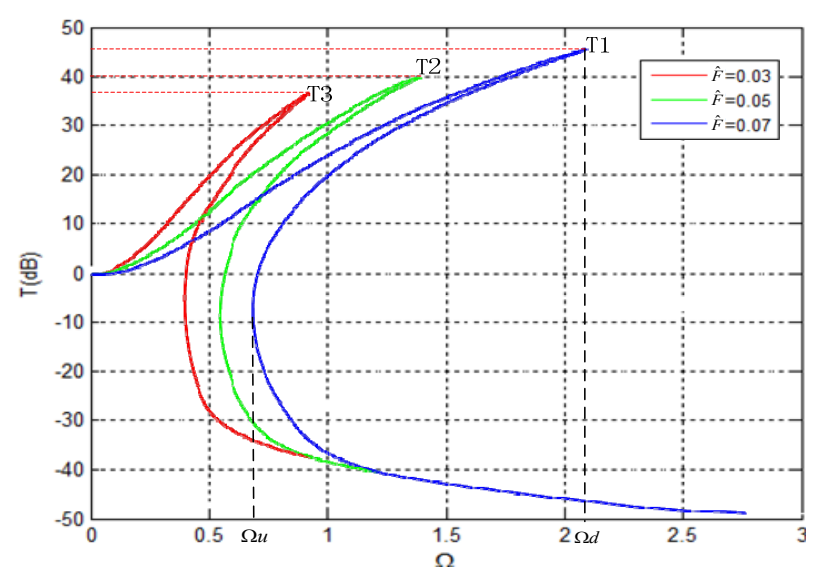

Fig. 9 Influence of exciting force amplitude on transmissibility for $\zeta=0.05, \gamma=5 / 18$.

In the jumping frequency domain, namely $\Omega_{d}<\Omega<\Omega_{u}$, the vibration isolator becomes two-state system. The upper branch and the lower branch are steady-state, while the middle branch is not steady-state. The jump-down frequency $\Omega_{\mathrm{d}}$ the jump-up frequency $\Omega_{\mathrm{u}}$ is the bifurcation point of the frequency response. To achieve available attenuation and broaden the frequency range of effective isolation, the jump-down frequency $\Omega_{\mathrm{d}}$ need to be decreased, as is shown in Fig. 9. As Fig. 9 shows, the relationship between the jump-up-and-down frequency and the amplitude of exciting force is direct proportion. As the blue curve shows in Fig. 9, the larger exciting force amplitude means the higher the jump-up-and-down frequency. Meanwhile, the peak transmissibility T1 corresponding to the largest exciting force amplitude is the highest compared with T2 and T3. Moreover, the frequency range of effective isolation is the narrowest because of increasing of the exciting force amplitude. Therefore, in order to achieve better effect of vibration isolation, we should select smaller amplitude of the exciting force.

\section{Conclusion}

This paper designs a novel mechanical adjustable isolator with quasi zero stiffness, obtains the condition the proposed system parameters should satisfy and analyzes the characteristics of system dynamics and the performance of vibration isolation. The following conclusions can be achieved through calculation and analysis. 1) The proposed vibration isolation system possesses simple structure, easy manufacturing and high static stiffness; 2) Distinguished from the common ones, when the bearing load changes, the proposed vibration isolation system can still maintain the zero stiffness characteristics nearby the static equilibrium position through adjusting thrust regulator; 3 ) Nearby the equilibrium position, the vibration isolation system can have excellent performance of vibration isolation under the small excitation amplitude, which is very suitable for vibration isolation with low and ultralow frequency.

\section{Acknowledgement}

This work was supported by the financial support from Natural Science Foundation of Anhui Province (Grant No. 1508085ME70).

\section{References}

[1] A. D. Shaw, S. A. Neild, M. I. Friswell. Relieving the effect of static load errors in nonlinear vibration isolation mounts through stiffness asymmetries. J. Sound Vib. 339 (2015) 84-98.

[2] X. T. Liu, Z. Y. Zhang, H. X. Hua. Characteristic of a novel low-requency isolator, J. Vib. Shock, 31 (5) (2012) 161-164.(In Chinese). 
[3] D. L. Xu, Q. P. Yu, J. X. Zhou. Study on Vibration Attenuation in Jumping Frequency Interval of Quasi-zero Stiffness Vibration Isolator. China Mech. Eng. 25 (2) (2014) 230-235. (In Chinese).

[4] W. S. Robertson, M. R. F. Kidner, B. S. Cazzolato, et al. Theoretical design parameters for a quasi-zero stiffness magnetic spring for vibration isolation. J. Sound Vib. 326 (1-2) (2009) 88-103.

[5] D. L. Platus. Negative-stiffness-mechanism vibration isolation systems. P. SPIE Int. Soc. Opt. Eng. 3786 (1999) 98-105.

[6] A. Carrella, M. J. Brennan, T. P. Waters, et al. On the design of a high-static-low-dynamic stiffness isolator using linear mechanical springs and magnets. J. Sound Vib. 315 (3) (2008) 712-720. 